\title{
Monitoreo del Herbazal del páramo una estrategia de medición del cambio climático en la Reserva de Producción de Fauna Chimborazo
}

\section{Grassland monitoring of the paramo a climate change measurement strategy in the Chimborazo Fauna Production Reserve}

\author{
Daniel Adrian Vistin Guamantaqui. ${ }^{1}$, Eduardo Antonio Muñoz Jácome. ${ }^{2}$ \& Guicela \\ Margoth Ati Cutiupala. ${ }^{3}$
}

Recibido: 10-01-2020 / Revisado: 25-02-2020 /Aceptado: 04-03-2020/ Publicado: 03-04-2020

\begin{abstract}
.
DOI: https://doi.org/10.33262/cienciadigital.v4i2.1195

The Chimborazo Fauna Production Reserve (RPFCH) is one of the most important protected areas in Ecuador whose fundamental objective is the preservation of the fauna and flora of the Andes, this work was carried out with the purpose of monitoring the grassland of the paramo, which is One of the eight plant formations that the reserve has to evaluate the changes that this altimontano ecosystem undergoes due to climate change, 128 orthophotos from the entire study area were used and with the help of the Arc Gis 10.1 software, everything related to cartography, for statistical analysis, the formulas of the Forest Management Manual of Andalusia were used, where the number of sample units was defined, working with $5 \%$ error and $95 \%$ certainty, optimal plots were used where the size of the approximate sample to estimate $\mathrm{p}$ with a limit of B for the estimation error, to inventory the flora the GLORIA methodology was used by the survey of four quadrants of $1 \mathrm{~m} 2 \times 1 \mathrm{~m} 2$ in 10 plots where the wealth, abundance, dominance and IVIE were established, the results of which were tabulated by the PAST 4.0 software, the sampling effort was validated with the help of the Eco Sim software, it was specified that the Poaceae, Asteraceae and Apiaceae families are the most dominant, alpha diversity indicated a value of 0.95 for the Simpson index and for
\end{abstract}

\footnotetext{
1 Escuela Superior Politécnica de Chimborazo, Facultad de Recursos Naturales, Proyecto MARERUS. Riobamba, Ecuador. daniel.vistin@espoch.edu.ec

${ }^{2}$ Escuela Superior Politécnica de Chimborazo, Facultad de Recursos Naturales, Proyecto MARERUS. Riobamba, Ecuador. emunoz@espoch.edu.ec

3 Escuela Superior Politécnica de Chimborazo, Facultad de Recursos Naturales, Proyecto MARERUS. Riobamba, Ecuador. guicela.ati@espoch.edu.ec
} 
Shannon Wiener of 3.31 high values for biological diversity, a value of $0.67 \%$ in similarity Floristics (beta) Due to the large presence of Calamagrostis intermedia, the Correspondence Analysis determined two ecological niches with 23 species, adding $51.05 \%$ of the total of sampled individuals, indicating that these are the most susceptible to disappear due to global warming.

Keywords: Paramo grassland, Climate change, Monitoring, Ecosystem, Altimontano.

\section{Resumen.}

La Reserva de Producción de Fauna de Chimborazo (RPFCH) es una de las áreas protegidas más importantes del Ecuador cuyo objetivo fundamental es la preservación de la fauna y la flora altoandina, este trabajo se efectuó con el propósito de monitorear el Herbazal del páramo que es una de las ocho formación vegetales que posee la reserva para evaluar los cambios que sufre este ecosistema altimontano debido al cambio climático, se utilizó 128 ortofotos de toda el área de estudio y con la ayuda del software Arc Gis 10.1 se geo proceso todo lo referente a la cartografía, para el análisis estadístico se utilizó las fórmulas del Manual de Ordenación de Montes de Andalucía donde se definió el número de unidades muestrales, se trabajó con un $5 \%$ de error y $95 \%$ de certeza se utilizó parcelas óptimas donde el tamaño de la muestra aproximado para estimar $p$ con un límite de $B$ para el error de estimación, para inventariar la flora se utilizó la metodología GLORIA mediante el levantamiento de cuatro cuadrantes de 1 $\mathrm{m}^{2}$ x $1 \mathrm{~m}^{2}$ en 10 parcelas donde se estableció la riqueza, abundancia, dominancia e IVIE cuyos resultados se tabularon el software PAST 4.0, el esfuerzo de muestreo se validó con la ayuda del software Eco Sim se precisó que las familias Poaceae, Asteraceae y Apiaceae son las más dominantes, alfa diversidad indicó un valor de 0,95 para el índice de Simpson y para Shannon Wiener de 3,31 valores altos para diversidad biológica, un valor de $0,67 \%$ en la similaridad florística (beta) debido a la gran presencia de Calamagrostis intermedia, el Análisis de correspondencia determinó dos nichos ecológicos con 23 especies sumando un 51,05\% del total de individuos muestreados indicando que estos son los más susceptibles a desaparecer por el calentamiento global.

Palabras claves: Herbazal del páramo, Cambio climático, Monitoreo, Ecosistema, Altimontano.

\section{Introducción.}

Ecuador es considerado uno de los 17 países megadiversos del mundo (Aguirre et al, 2017), debido a su sorprendente biodiversidad comprendida en una reducida superficie $0,2 \%$ del planeta, de esta área aproximadamente el $20 \%$ del territorio nacional 5 millones de hectáreas se enmarcan en la categoría de protección de acuerdo con la Legislación Ambiental Nacional por Constitución de la República, estas conforman el Sistema Nacional de Áreas Protegidas 
(SNAP) conocido como Patrimonio de Áreas Naturales del Estado (PANE), distribuidas en todo el territorio continental e insular, albergan una importante riqueza biológica, servicios ecosistémicos de los cuales se benefician tanto las poblaciones urbanas como rurales, una riqueza paisajística que permite el turismo y la recreación en parte de ellas y por su importancia ecológica trascienden fronteras que son reconocidas a nivel internacional (MAE, 2017).

La importancia de gestionar de forma sostenible la biodiversidad se sustenta, en su rol en mantener procesos ecosistémicos saludables, como recurso estratégico con valor económico realizado y potencial, y como legado para las futuras generaciones en Ecuador (Cuesta et al, 2015). En este contexto, es necesario pensar en estrategias de conservación, manejo sostenible, monitoreo y rehabilitación en paisajes más amplios que soportan diversos usos de la tierra, a menudo no compatibles con la persistencia de la biodiversidad.

En este sentido este estudio se enfoca en el ecosistema Herbazal de páramo el cual en América latina está distribuido dentro de la zona húmeda de los Andes ecuatoriales entre $11^{\circ} \mathrm{N}$ y $8^{\circ} \mathrm{S}$ de latitud, principalmente en Venezuela, Colombia, Ecuador y el norte de Perú, así también en algunas zonas de Costa Rica y Panamá (Hofstede et al, 2014). El páramo es uno de los ecosistemas de alta montaña más ricos del mundo con una gran diversidad y endemismo, su importancia biogeográfica, evolutiva, ecológica y económica hacen de este ecosistema un sitio único en el mundo y las especies que se han adaptado a él no se encuentran en ningún otro lugar, razón por la cual se considera un ambiente sumamente frágil (Sklenár \& Balslev, 2005). En el Ecuador el páramo incluye ecosistemas dominados por pajonales localizados en la franja comprendida entre el bosque montano alto y el límite inferior de la nieve (Llambí et al, 2012) aproximadamente desde los $3700 \mathrm{~m}$ en la Cordillera Oriental y 3 $400 \mathrm{~m}$ en la Occidental de los Andes (Castañeda \& Montes, 2017). Esta formación vegetal está presente en catorce de cincuenta y un áreas protegidas del Ecuador que comprende el 5 $\%$ del territorio nacional continental (Hofstede et al., 2014).

Los páramos proporcionan un enorme servicio ambiental al ser grandes abastecedores de agua para consumo en las comunidades localizadas a sus alrededores y en ciudades grandes (Buytaert \& Ablan, 2014; García et al, 2019)Además, indirectamente cientos de miles de personas se benefician de los páramos al recibir agua para riego y/o para generar energía eléctrica sin contar también el beneficio ecosistémico de captura de carbono (Cunalata et al, 2013). Los páramos también tienen gran importancia como atractivo turístico y como bancos genéticos de plantas andinas que han sido cultivadas durante milenios. Lamentablemente el páramo como tantos otros ecosistemas tropicales, está siendo destruido por las actividades humanas y el calentamiento global. Esto hace que califique este ecosistema como de alta prioridad para la conservación (Díaz et al, 2005).

La Reserva de Producción de Fauna de Chimborazo se encuentra ubicada en los Andes septentrionales, de acuerdo con la división política se encuentra entre las provincias de 
Tungurahua, Chimborazo y Bolívar. La RPFCH es parte del Sistema Nacional de Áreas Protegidas (SNAP) desde 1987 como una de las áreas más importantes del país ya que cumple la función de recuperación de dos especies emblemáticas del país, Según el Ministerio del Ambiente el monitoreo es crucial para entender la dinámica que provoca el cambio ambiental global en la biodiversidad a largo plazo en ecosistemas de alta montaña ya que en estos ecosistemas es donde más se hace visible las afectaciones en la composición, estructura, función y dinámica de los ecosistemas paramunos ( MAE, 2014; Urgiles et al, 2018).

El herbazal de páramo es una formación vegetal densa dominada por gramíneas en forma de macollas que alcanzan o son mayores a $50 \mathrm{~cm}$ de altura, este ecosistema rodea la mayor extensión de los entornos altimontanos en el Ecuador, en la RPFCH que es objeto de estudio está conformado por 7470,31 ha que representa el 14,07 \% del área total de esta reserva (Paula et al, 2018), en el Ecuador este bioma está presente en la cordillera de los Andes desde la provincia de Carchi en el Norte hasta la provincia del Carchi en el Sur (Beltrán et al., 2009). Es propio del piso montano alto superior y generalmente se lo encuentra en valles glaciares, laderas de vertientes disectadas y llanuras subglaciares sobre los $3400 \mathrm{msnm}$. Según los resultados obtenidos en el ecosistema de herbazal de páramo herbáceo, tenemos básicamente como forma de vida dominante las "mocollas" formadas por la denominada "paja de páramo", dando lugar a grandes extensiones de los llamados "pajonales". La especie con mayor presencia son Calamagrostis intermedia y Agrostis perennans cuyas coberturas vegetales dominan en un $75 \%$ formando comunidades monotípicas.

En la RPFCH el estado de conservación de este ecosistema se ve afectado principalmente debido a actividades antrópicas (quema de pajonales, sobrepastoreo, introducción de especies exóticas y avance de la frontera agrícola) por parte las comunidades que se asientan dentro y en la zona de amortiguamiento del parque, en cuanto a la diversidad en estos ecosistemas los valores indican una baja diversidad, no obstante teniendo en consideración que la latitud y altitud son los factores determinantes de la diversidad (Vistin \& Barrero, 2017) esta formación vegetal se la considera rica al presentar un sinnúmero de especies endémicas adaptadas a las condiciones adversas donde habitan especialmente en forma de rosetas y rastreras.

Bajo esta perspectiva, el desarrollo de esta investigación permite obtener información concreta para fortalecer las redes globales de monitoreo ecológico. La presente investigación forma parte del Proyecto "Medidas ante los riesgos que afrontan los ecosistemas de la reserva de producción de fauna Chimborazo frente al cambio de uso de suelo" (MARERUS ESPOCH) del ecosistema Herbazal de páramo de la RPFCH el cual busca generar medidas de conservación fundamentadas en el entendimiento de esta formación vegetal además de sus efectos relacionados al cambio climático y su impacto en la biodiversidad. Así, en el presente trabajo se genera información sobre la diversidad florística del ecosistema para el monitoreo a mediano y largo plazo de esta comunidad vegetal. 


\section{Metodologia.}

\section{Àrea de estudio}

La investigación se desarrolló en el ecosistema Herbazal del páramo, las 10 parcelas de monitoreo se encuentran localizadas en la parroquia Pilahuin província de Tungurahua dentro de la Reserva de producción de fauna Chimborazo.Este lugar tiene una extensión de 58560 ha (MAE, 2014) de las cuales el Herbazal de páramo està presente en 7 470,31 ha (14,07 \%) distribuido en un rango altitudinal que va desde los 3800 y $4200 \mathrm{msnm}$ este ecosistema se caracteriza en algunos sitios por ser una confluencia entre los ecosistemas Arbustal siempreverde y Herbazal del páramo que comprende una fisonomía arbustiva y herbácea la cual se la cataloga como una zona de alto endemismo.

La zona de estudio se localizan en las coordenadas 731400 E y 9851100 S (en metros) a una altitud $4200 \mathrm{msnm}$, en cuanto a los factores de diagnóstico de la zona presenta una fisonomía herbácea y en algunas zonas arbustiva un bioclima pluviestacional, ombrotipo húmedo y una fenología siempreverde y por la característica geográfica es una zona de no inundabilidad debido a la pendiente, este sitio se caracteriza por poseer una temperatura promedio máximo de $8,81{ }^{\circ} \mathrm{C}$ en las estribaciones oriental y occidental de la reserva (INAMHI, 2019).

La temperatura mínima registrada en la $\mathrm{RPFCH}$ es de $-4,80{ }^{\circ} \mathrm{C}$ en el mes de diciembre y la máxima de $11,40{ }^{\circ} \mathrm{C}$ en el mes de noviembre, en cuanto a la precipitación es frecuente en los días más fríos y húmedos que ocurra en forma de nieve o escarcha en las zonas más altas, se registra un promedio anual de $998 \mathrm{~mm}$ que varía entre $809 \mathrm{~mm}$ en las zonas menos lluviosas y los 1300 mm en las zonas más húmedas (MAE, 2013).

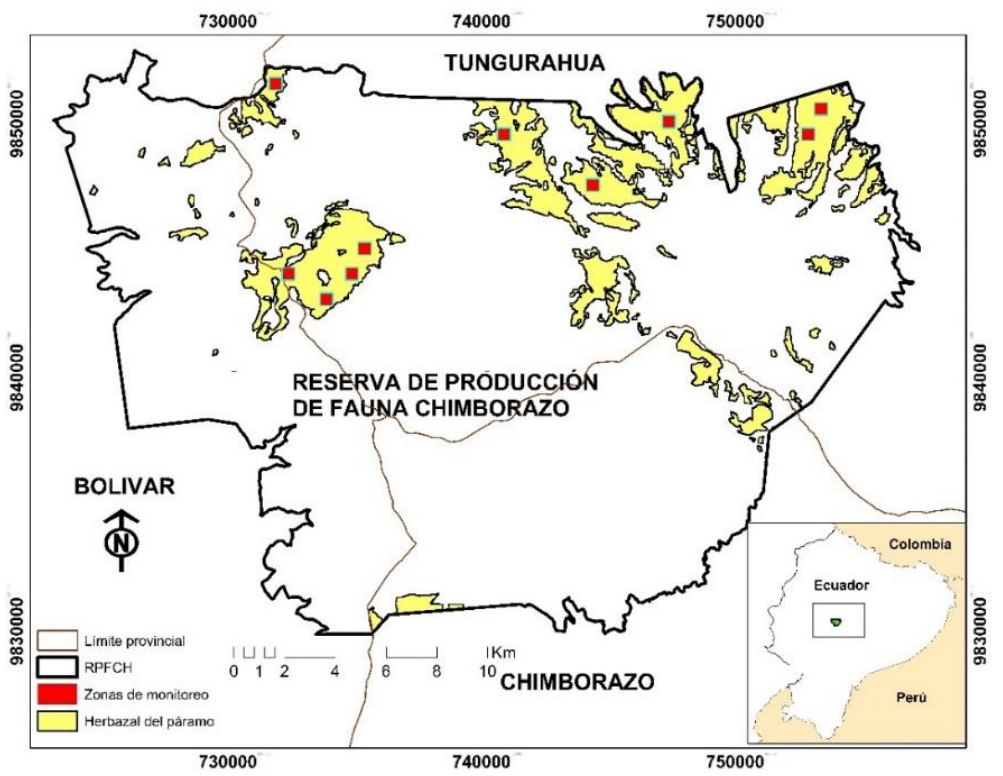

Figura 1. Ubicación de las parcelas de monitoreo del en la RPFCH Fuente: Elaboración propia. 


\section{Análisis estadístico}

Para determinar el número de unidades muestrales se elaboró celdillas de 500 X $500 \mathrm{~m}^{2}$ en el ecosistema Herbazal del páramo para esto se utilizó el software Arc Gis 10.1 con la ayuda de la herramienta (Hawths Tools - Sampling Tools - Create Vector Grid) se construyó 30 celdillas considerando solo las que no estaban en una zona de ecotono ecológico y en la zona de estricta conservación para evitar el efecto de borde a la hora de la toma de datos en campo.

Se tomó en consideración el criterio de (Rodriguez et al., 2004) que propone en el Manual de Ordenación de Montes de Andalucía para determinar el número de unidades muestrales, en cuanto al error se trabajó con un $5 \%$ y $95 \%$ de certeza para ello se utilizó parcelas óptimas donde el tamaño de la muestra aproximado para estimar $p$ con un límite de $B$ para el error de estimación, determinándose el número de puntos donde se recolectarían los datos para su análisis (Figura 1) donde se utilizó la siguiente ecuación:

$$
n=\frac{\sum_{i=l}^{L} N_{i}^{2} p_{i} q_{i} / a_{i}}{N^{2} D+\sum_{i=l}^{L} N_{i}^{2} p_{i} q_{i}}
$$

Donde:

$a_{i}=$ es la fracción de observaciones fijadas al estrato

$i, p_{i}=$ es la proporción poblacional para el estrato

$i$ y $D=B^{2} / 4$

Tabla 1. Determinación del número de unidades muestrales del ecosistema Herbazal del Páramo

\begin{tabular}{ll}
\hline Formación Vegetal & herbazal del páramo \\
\hline Código & $\mathrm{HsSnO} 2$ \\
$\mathbf{N}$ & 30 \\
$\mathbf{p}$ & 0,5 \\
$\mathbf{q}$ & 0,5 \\
$\mathbf{a i}$ & 0,04 \\
$\mathbf{\Sigma}$ num & 5880 \\
$\mathbf{\Sigma}$ den & 5700 \\
$\mathbf{n i}$ & 9,87 \\
$(\mathbf{n})$ redon & $\mathbf{1 0}$ \\
\hline
\end{tabular}

La metodología que se utilizó para la inventariación de la flora es la que propone Pauli et al, (2015) para el proyecto GLORIA en el territorio europeo, por tal motivo se hicieron modificaciones para que se ajusten al contexto de Herbazal en el Ecuador, esto permitió levantar la información de forma precisa y fuertemente respaldada ya que esta metodología precisa medir los cambios en la vegetación debido al cambio climático, con la ayuda de 128 ortofotos de toda la Reserva se estableció los puntos de monitoreo y se comprobó en campo 
si se trataba de la misma tipología además del estado de conservación. En cada punto de muestreo se instaló un cuadrante de $5 \times 5 \mathrm{~m}$ con un área de $250 \mathrm{~m}^{2}$ que se utilizó para el muestreo, la recolección de datos se hizo en los extremos de cada parcela de $1 \mathrm{~m}^{2}$ evitando de esta manera que se alteren los sitios donde se recolectaran los datos.

Con la ayuda de un armazón de madera de 1 x $1 \mathrm{~m}$ con un enrejado hecho por hilos finos que demarcan 100 celdillas se obtuvo la información referente a la riqueza mientras que para la abundancia y dominancia los datos fueron tomados en porcentaje de acuerdo a su cobertura en los cuadrantes sobre un $400 \%$. Se determinó la densidad relativa (DR), frecuencia relativa (FR) y el índice de valor de importancia (IVI) para cada una de las especies registradas dentro del área de monitoreo con el objetivo de conocer la composición de la comunidad vegetal, por otro lado, mediante un Análisis de Correspondencia (CA) se analizará la distribución espacial para establecer posibles asociaciones entre ellas.

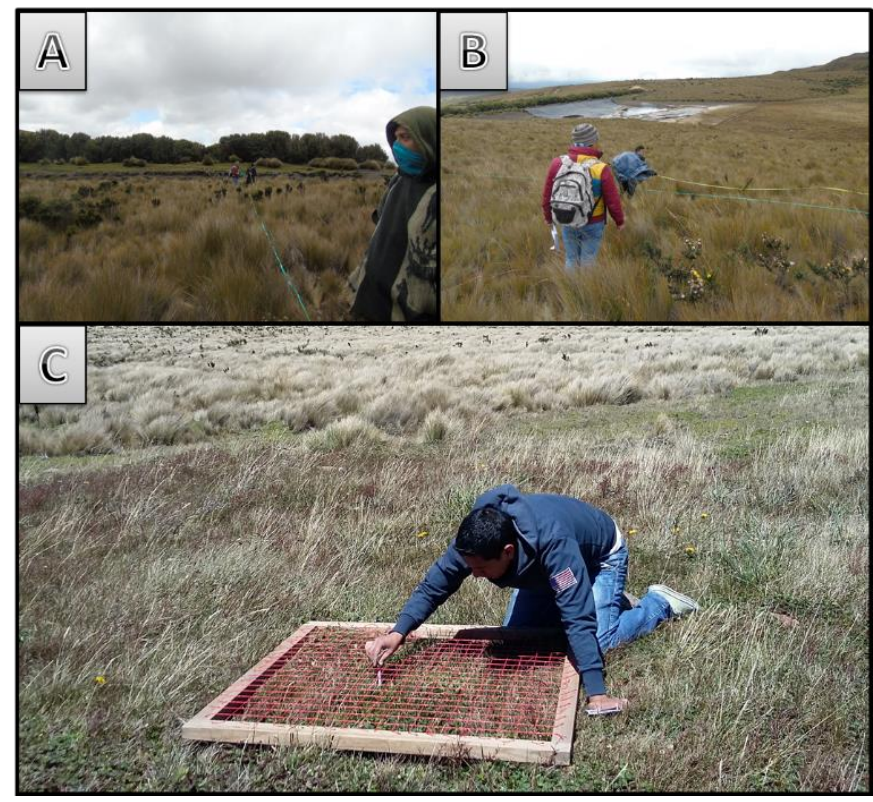

Figura 2. Fotografías ilustrativas del Herbazal del páramo. A) Zonificación de la formación vegetal, B) Selección de los sitios de muestreo, C) Inventariación de las especies.

\section{Resultados}

\section{Validación del esfuerzo de muestreo}

De acuerdo con los datos calculados mediante el software Eco Sim teniendo en cuenta la abundancia, riqueza, límite inferior y límite superior con un porcentaje de confiabilidad del $95 \%$, la tendencia de la curva de rarefacción obtiene la asíntota con un valor de 500 individuos por lo que se considera que el muestreo fue suficiente para representar la composición florística y la diversidad biológica de la comunidad. 


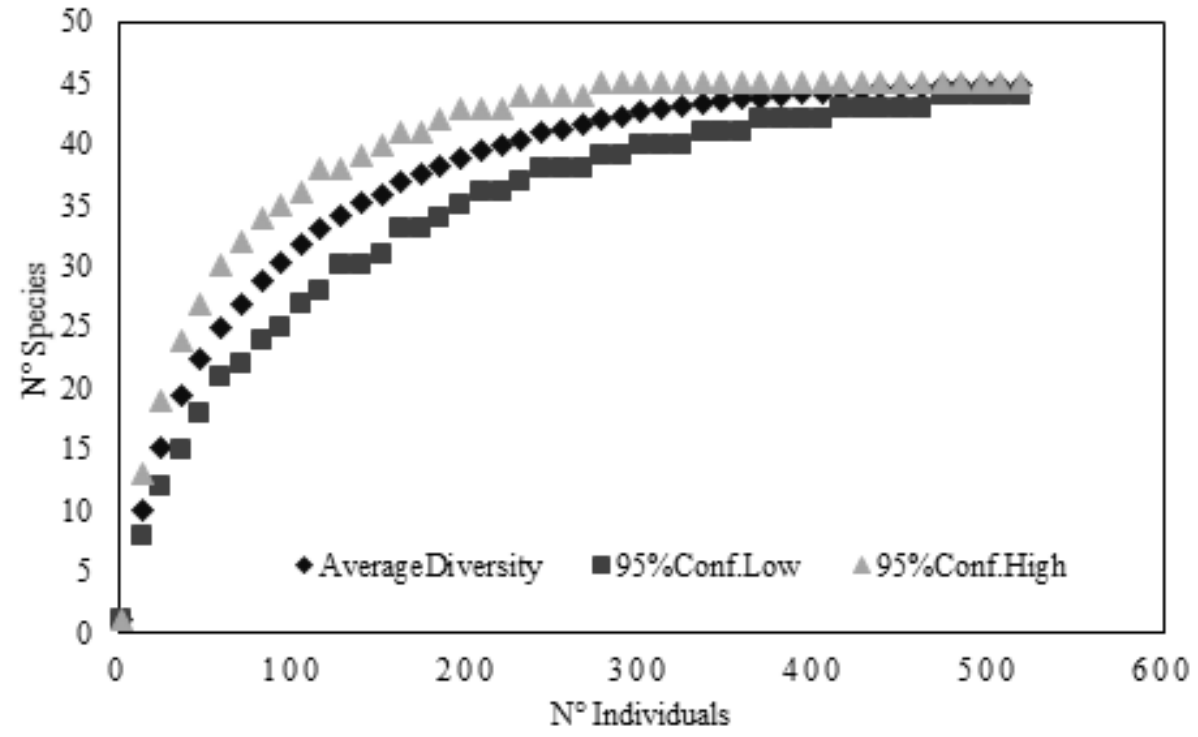

Figura 3. Curva de rarefacción para validar el esfuerzo de muestreo del Herbazal del Páramo

\section{Composición florística y parámetros ecológicos}

En el estudio de la formación vegetal se encontró un total de 551 individuos de 23 familias y 29 géneros distribuidas en los diez sitios de muestreo dentro de la RPFCH, del total de familias vegetales encontradas las que presentaron el mayor número fueron Asteraceae y Apiaceae con 14 y 3 especies respectivamente, en estos momentos no es posible un análisis cronológico preciso de la flora del Herbazal de páramo pero si se pueden observar tendencias con base en la obra de Santa Cruz et al, (2019) en estudios en Perú en condiciones edáficas y climáticas con óptimo holártico similares donde afirma que las familias más representativas de estos ecosistemas son Asteraceae y Poaceae.

La especie Calamagrostis intermedia funge como la más frecuente ya que fue registrada en las cuarenta subunidades de muestreo de $1 \mathrm{~m}^{2}$ seguida de Lachemilla orbiculata presente en 32 de los cuarenta sitios muestreados sumando entre las dos un total del $92 \%$ considerándolas como especies representativas de esta formación vegetal.

Tabla 1. Índice de valor de importancia ecológica del Herbazal del Páramo (RPFCH)

\begin{tabular}{lcccc}
\hline \multicolumn{1}{c}{ ESPECIE } & FR & DR & AR & IVIE \\
\hline Calamagrostis intermedia (J. Presl) Steud & $\mathbf{0 , 0 8}$ & $\mathbf{5 8 , 4 2}$ & $\mathbf{1 0 , 7 1}$ & $\mathbf{6 9 , 2 0}$ \\
Lachemilla orbiculata (Ruiz \& Pav.) Rydb. & $\mathbf{0 , 0 7}$ & $\mathbf{6 , 9 3}$ & $\mathbf{9 , 8 0}$ & $\mathbf{1 6 , 8 0}$ \\
Geranium sibbaldioides Benth. & 0,03 & 0,99 & 2,90 & 3,93 \\
Hypochaeris sessiliflora Kunth. & 0,02 & 0,25 & 1,09 & 1,36 \\
Eryngium humile Cav. & 0,04 & 0,74 & 5,63 & 6,41 \\
Diplostephium ericoides (Lam.) Cabrera & 0,04 & 3,22 & 2,18 & 5,44 \\
Werneria pygmaea Gillies ex Hook. \& Arn & 0,04 & 1,24 & 6,90 & 8,17 \\
\hline
\end{tabular}


ISSN: 2602-8085

Vol. 4, $\mathrm{N}^{\circ}$ 1, p. 32-47, enero-marzo, 2020

Valeriana microphylla Kunth

$\begin{array}{llll}0,02 & 0,74 & 1,63 & 2,40\end{array}$

Huperzia crassa (Humb. \& Bonpl. Ex Willd.) Rothm.

0,03

0,99

2,18

3,20

Lachemilla hispidula (L. M. Perry) Rothm.

0,03

0,25

1,81

2,09

Stellaria serpyllifolia Willd. ex D.F.K. Schltdl.

0,02

1,49

5,26

6,77

Befaria resinosa

Lupinus microphyllus Desr. N. v.

0,02

0,50

0,54

1,06

Peperomia hispidula

0,02

0,74

2,54

3,31

Aetheolaena involucrata (Kunth) B. Nord.

0,02

1,49

4,36

5,87

Elaphoglossum ovatum (Hook. \& Grev.) T. Moore

0,02

0,25

0,91

0,02

1,24

2,72

1,17

Gynoxys acostae Cuatrec.

0,02

1,98

0,73

3,98

Clinopodium nubigenum (Kunth)

0,03

2,72

7,62

2,73

Miconia salicifolia (Bonpl. ex Naudin) Naudin

0,02

0,50

0,36

10,38

Blechnum loxense (Kunth) Hook. ex Salomon

0,02

0,25

0,73

0,87

Monticalia empetroides (Cuatrec) C. Jeffrey

0,02

0,74

0,54

0,99

Cotula mexicana (DC.) Cabrera

0,02

0,74

4,90

1,30

Hypericum laricifolium Juss.

Pernettya prostrata (Cav.) DC.

0,02

0,99

0,73

5,67

0,02

0,25

0,73

1,73

Thelypteris retrorsa (Sodiro) A. R. Sm.

Taraxacum officinale

0,02

0,50

1,63

0,99

0,02

0,50

1,27

0,02

0,25

0,73

2,15

Vicia andicola Kunth

Halenia weddelliana Gilg.

0,03

0,99

4,90

0,02

0,50

1,63

0,02

1,73

0,91

0,02

0,25

0,54

0,01

0,25

0,54

0,02

0,50

0,54

0,02

0,50

1,81

0,01

0,74

0,54

0,0

0,50

0,18

0,01

0,25

0,18

$0,01 \quad 0,25$

0,91

0,01

0,50

0,36

0,01

0,25

0,54

0,01

0,50

0,54

0,01

0,25

0,91

0,01

0,25

0,36

1,79

0,99

Geranium maniculatum H.E. Moore

0,01

0,25

0,54

$0,01 \quad 0,74 \quad 2,54$

5,92

2,15

Valeriana plantaginea Kunth

$0,01 \quad 1,98$

0,36

2,66

0,81

0,80

1,06

2,33

1,30

0,68

0,44

1,16

0,87

0,80

1,05

1,16

0,62

0,80

Trifolium repens L

3,29

Fuente: Elaboración propia. 
Las especies ecológicamente más dominantes resultaron ser Calamagrostis intermedia y Lachemilla orbiculata ya que estas son las más conspicuas lo cual se puede observar en toda el área de estudio, esto se corrobora con resultados similares efectuados por Beltrán et al, (2009) en páramos del volcán Carihuairazo en cotas de 3800 a 4000 msnm asegurando que se observa la predominancia de la alianza de pajonales y almohadillas de Plantago $s p$, Calamagrostis sp, Werneria sp. y Lachemilla sp siendo sus especies diagnósticas Plantago rigida, Calamagrostis intermedia, Werneria pygmaea y Lachemilla orbiculata.

En cuanto al resultado del índice de Simpson como medida de dominancia donde las especies comunes tienen el mayor peso con respecto a las denominadas especies raras expresada la probabilidad de extraer del Herbazal del páramo dos individuos al azar de la misma especie se obtuvo un valor de 0,95 por lo que se considera como alta la medida de dominancia, de igual manera Caranqui et al, (2016) reporta valores similares en zonas de páramo para este índice los cuales fluctúan de 0.17 - 0.79 corroborando la variabilidad florística de la reserva.

Teniendo en consideración la riqueza de especies y su abundancia se determinó el índice de Shannon Wiener $\left(\mathrm{H}^{\prime}\right)$ con el objetivo de cuantificar la biodiversidad específica cuyo resultado fue de 3,31 por lo que se considera este valor como alto en diversidad en esta formación vegetal.

Para dejar bien asentado el estudio florístico se midió la proporción de la diversidad observada con relación a la máxima diversidad esperada para determinar si las especies son igualmente abundantes en los 10 sitios de muestreo para esto se lo efectuó con ayuda del índice de Pielou en donde resulto un valor de 0,52 esto se lo puede denotar más detalladamente en el análisis del IVIE (Tabla 1) donde se observa que las especies más abundantes y más frecuentes corresponden a Calamagrostis intermedia, Lachemilla orbiculata, Werneria pygmaea, Stellaria serpyllifolia, Clinopodium nubigenum, Eryngium humile mientras que el resto de especies en las zonas muestreadas aparecen con menor frecuencia.

Dentro de la diversidad florística a más de la altitud intervienen otros factores, como velocidad e intensidad del viento, pendiente, composición del suelo, radiación, entre otros, que generan condiciones muy particulares dentro de cada sitio de monitoreo así observándose en algunas zonas que su composición cambia ligeramente en el noreste de la reserva en las estribaciones del volcán Carihuairazo ya que debido a la topografía aparecen especies nuevas arbustivas las cuales se asocian con otras especies aumentado la riqueza especifica.

\section{Similaridad florística}

La similaridad florística de las comunidades muestreadas se evaluó mediante el índice de Jaccard (J), de forma exclusivamente cualitativo y no considerando el grado de participación de cada especie en la dominancia ecológica, donde el valor más cercano a 1 indica mayor semejanza (Magurran, 2004). Este índice se define mediante la ecuación: Índice de Jaccard: 
$\mathrm{Jij}=\mathrm{C} / \mathrm{S} 1+\mathrm{S} 2-\mathrm{C}$ Donde: $\mathrm{S} 1=\mathrm{N}^{\circ}$ de especies presentes en la zona $1 \mathrm{~S} 2=\mathrm{N}^{\circ}$ de especies presentes en la zona $2 \mathrm{C}=\mathrm{N}^{\circ}$ de especies que están presentes en las dos muestras.

Este es el cociente de la intersección entre la suma de todo lo no común del Herbazal de páramo. Es al contrario del índice de inclusión o índice simétrico donde sus valores oscilan entre 0 y 1 . Cuando la intersección es nula, Jij $=0$, y cuando los conjuntos son idénticos, Jij $=1$ bajo este análisis se establece el dendrograma de similaridad.

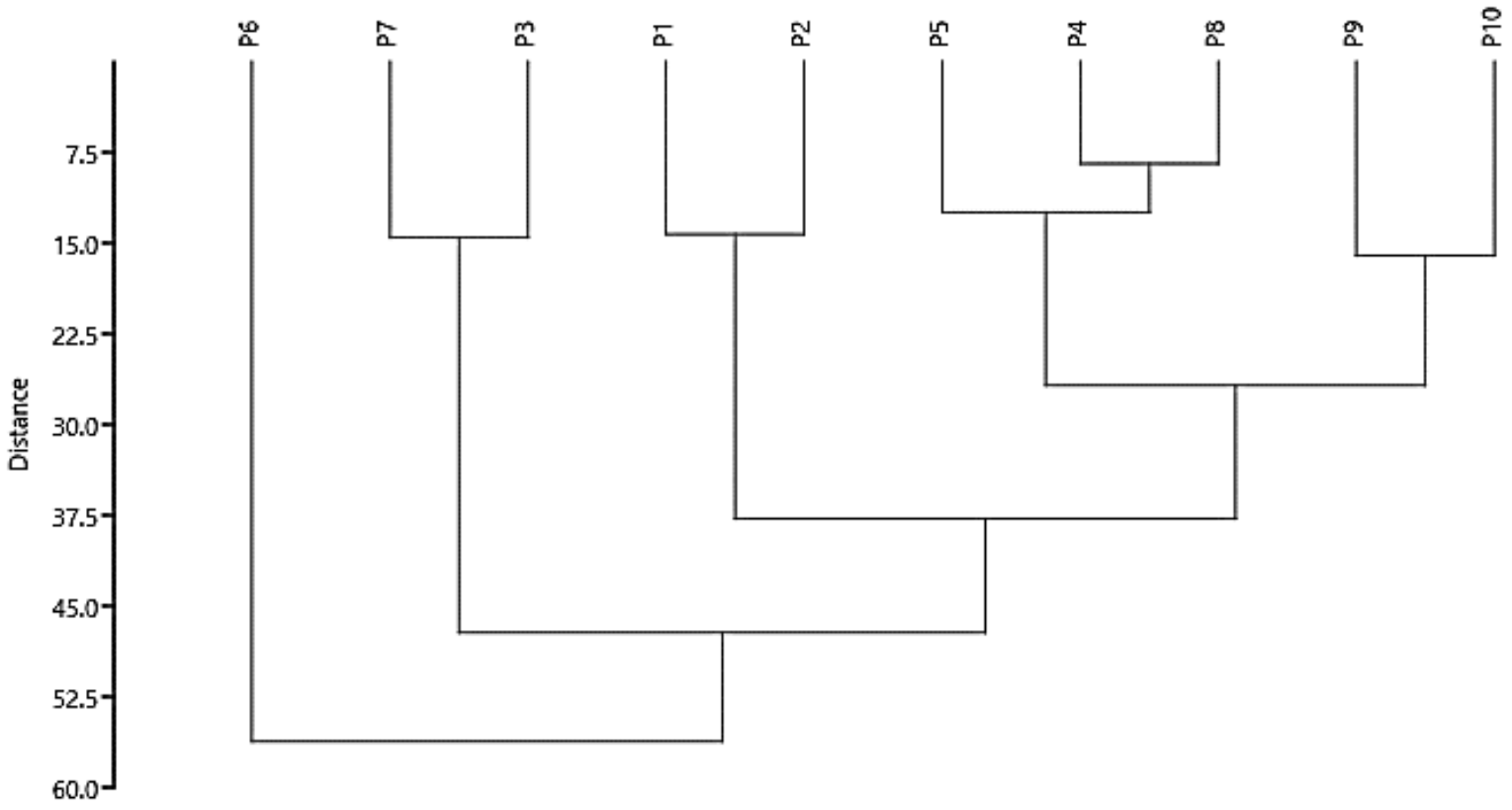

Figura 4. Similaridad florística del Herbazal del páramo

La similaridad florística se basa en la incidencia de las especies en cada ambiente (presencia o ausencia) y el resultado se puede visualizar como la proporción o porcentaje de especies compartidas, permitiendo conocer en forma detallada la semejanza entre comunidades florísticas que previamente han sido definidas. La formación vegetal Herbazal del páramo presento un índice de similaridad de 0,67 debido a que 38 de las 47 especies estuvieron presentes en los 10 sitios de muestreo.

La primera agrupación la conforma la parcela (P6) debido a que solo en esta se encontró la especie Carex pichinchensis, el segundo grupo está conformado por la parcela (P7, P3) ya aquí se registró especies en común Stellaria serpyllifolia, Peperomia hispidula y Elaphoglossum ovatum como las más dominantes, de igual manera el tercer grupo lo conforma la parcela P1 y P2 ya que comparten especies en común como Werneria pygmaea, Hypochaeris sessiliflora y Eryngium humile, de igual manera el grupo 4 lo conforman las parcelas (P5, P4, P8) con la singularidad de que en estas se registró a Lachemilla hispidula, y el último grupo está conformado por las parcelas (P9, P10) donde la presencia de las especies Clinopodium nubigenum y Halenia weddelliana las hace tener relación de 
similaridad, todas las parcelas inventariadas tienen a Calamagrostis intermedia y Lachemilla orbiculata como especies comunes más abundantes, dominantes y más frecuentes.

\section{Análisis de correspondencia (CA) de la distribución espacial de especies}

Según la afirmación de Joly \& Myers (2001) la variabilidad espacial de la biodiversidad es la respuesta a una serie de procesos que interactúan a escalas temporales y especiales como movimientos tectónicos en eras geológicas pasadas o glaciaciones que han influido en la aparición y desaparición de las especies, por tal motivo para actividades de monitoreo del Herbazal del páramo es necesario analizar la distribución espacial para comprender mejor como estas forman nichos ecológicos según el sitio donde se encuentran y cuál es la dinámica espacio temporal.

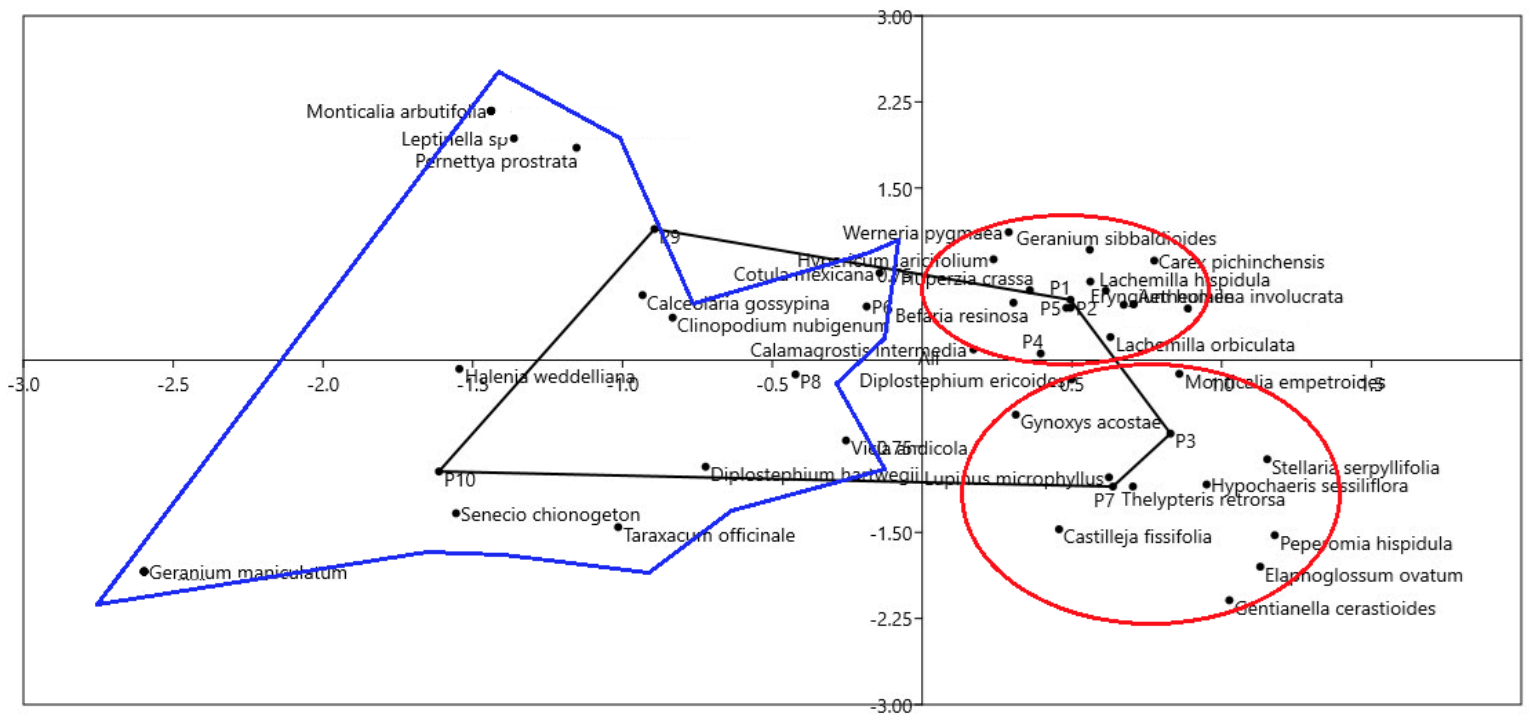

Figura 5. Análisis de distribución espacial del Herbazal del páramo de la RPFCH

El análisis en base al gradiente altitudinal demuestra que las especies Calamagrostis intermedia y Lachemilla orbiculata tienen una tendencia de estabilidad ecológica dentro de la formación vegetal ya que en la gráfica estas se aproximan al valor de cero en ambos axis debido a que estas poseen un rango de adaptabilidad climática más extensa. Mediante este estudio se determinó dos nichos ecológicos no tan marcados donde el polígono tiende a estrecharse (axis 1 y 2) explicando la influencia de la variable altitud en la presencia o no de los individuos, el primero está representado por 13 especies 27,65\% y el segundo por 11 especies $2340 \%$ lo cual indica que la mitad de estas aparecen en lugares con una altitud similar, mediante estos resultados se establece las especies que servirán para futuros estudios de monitoreo en esta formación vegetal ya que a medida que aumenta la temperatura del planeta se podrá medir si estas colonizaron nuevas zonas a mayor altitud mediante la dinámica del cambio de uso de suelo. 


\section{Conclusiones.}

- La riqueza florística reportada en el presente estudio contiene una muestra representativa de la diversidad vegetal del ecosistema Herbazal del páramo de la RPFCH que está integrada por 47 especies 29 géneros y 23 familias denotando su alta diversidad biológica pese a que este estudio se efectuó en cotas sobre los $4000 \mathrm{msnm}$.

- Los índices de diversidad presentan valores con tendencia de medios a altos connotándose que la dominancia y abundancia tienen correlación, no sucede lo mismo con la frecuencia ya que el valor oscila de bajo a medio debido a que si bien es cierto que la mayoría de las especies están presentes en un gran número de parcelas se las encuentra con una menor frecuencia debido a que Calamagrostis intermedia y Lachemilla orbiculata tienden a monopolizar estas zonas como las más dominantes.

- Mediante el análisis de correspondencia canónico para determinar la distribución espacial se estableció que de las 47 especies registradas 23 de ellas forman dos nichos ecológicos más o menos definidos intrínsecamente con la variable altitud, indicando que estas son las más susceptibles a desaparecer debido al calentamiento global no así con las restantes que se disgregan en un mayor gradiente altitudinal dentro de la reserva.

\section{Referencias bibliográficas.}

Aguirre, Z., Mendoza, N., \& Muñoz, J. (2017). Biodiversidad de la provincia de Loja , Ecuador Biodiversity of the province of Loja , Ecuador. Arnaldoa, 24(2), 523-542.

Beltrán, K., Salgado, S., Cuesta, F., León-Yánez, S., Romoleroux, K., Ortiz, E., Cárdenas, A., \& Velástegui, A. (2009). Distribución espacial, sistemas ecológicos y caracterización florística de los páramos en el Ecuador Proyecto Páramo Andino y Herbario QCA. Quito. (EcoCiencia). www.ecociencia.org

Buytaert, W., \& Ablan, M. (2014). El páramo andino como productor y regulador del recurso agua. El caso de la microcuenca alta de la Quebrada Mixteque, Sierra Nevada de Mérida, Venezuela. January.

Caranqui, J., Lozano ${ }^{1}$, P., \& Reyes ${ }^{1}$, J. (2016). Composición y diversidad florística de los páramos en la Reserva de Producción de Fauna Chimborazo, Ecuador (Composition and diversity of High Andean in the Fauna Production Reserve Chimborazo, Ecuador). ENFOQUE, 1(April), 33-45. http://ingenieria.ute.edu.ec/enfoqueute/

Castañeda, A., \& Montes, C. (2017). Carbono almacenado en páramo andino. ENTRAMADO, 13(1), 210-221. https://doi.org/10.18041/entramado.2017v13n1.25112

Cuesta, F., Peralvo, M., Baquero, F., Bustamante, M., Merino-Viteri, A., Muriel, P., Freile, J., \& Torres, O. (2015). Áreas prioritarias para la conservación del Ecuador continental. In Ministerio de Ambiente, CONDESAN, Pontificia Universidad Católica del Ecuador, GIZ, 1-109.

Cunalata, C., Inga, C., Recalde, C., \& Echeverría, M. (2013). Determinación de carbono orgánico total presente en el suelo y la biomasa de los páramos de las comunidades del 
chimborazo y shobol llinllin en Ecuador. Boletín Del Grupo Español Del Carbón, 27, $10-13$.

Díaz, M., Navarrete, J., \& Suárez, T. (2005). Páramos hidrosistemas sensibles. Revista de Ingeniería, 22, 64-75.

García, V. J., Márquez, C. O., Isenhart, T. M., Rodríguez, M., Crespo, S. D., \& Cifuentes, A. G. (2019). Evaluating the conservation state of the páramo ecosystem: An objectbased image analysis and CART algorithm approach for central Ecuador. Heliyon, 5(10). https://doi.org/10.1016/j.heliyon.2019.e02701

Hofstede, R., Calles, J., López, V., Polanco, R., Torres, F., J, U., Vásquez, A., \& Cerra, M. (2014). Los Páramos Andinos ¿Qué sabemos? Estado de conocimiento sobre el impacto del cambio climático en el ecosistema páramo. UICN, Quito, Ecuador. In Tiempos de Crisis sistémica. https://doi.org/10.2307/j.ctvpv50bh.8

INAMHI. (2019). Boletín meteorológico 534 agosto 2019. http://www.serviciometeorologico.gob.ec/meteorologia/boletines/bol_men.pdf

Joly, K., \& Myers, L. (2001). Patterns of mammalian species richness and habitat association in Pennsylvania. Biological Conservation, 99, 253-260.

Llambí, L. D., Soto, A., Borja, P., Ochoa, B., Celleri, R., \& Bievre, B. (2012). Páramos Andinos: Ecologia, hidrología y suelos de páramos. In Los suelos del Páramo.

MAE. (2013). Metodología para la Representación Cartográfica de los Ecosistemas del Ecuador Continental. Subsecretaría de Patrimonio Natural. Quito, 1-106. http://www.ambiente.gob.ec/wpcontent/uploads/downloads/2013/03/Documento_Metodología_28_05_2012_v2_1.pdf

MAE. (2014). MAE (Ministerio del Ambiente del Ecuador) Actualización del plan de manejo de la Reserva de producción de Fauna Chimborazo, 1-297.

MAE. (2017). Estrategia Nacional de Biodiversidad 2015-2030, 1-225.

Magurran, A. E. (2004). Measuring Biological Diversity. Main Street, Malden, MA 021485020, USA. http://www.wiley.com/WileyCDA/WileyTitle/productCd0632056339.html

Paula, P. A., Zambrano, L., \& Paula, P. (2018). Análisis Multitemporal de los cambios de la vegetación, en la Reserva de Producción de Fauna Chimborazo como consecuencia del cambio climático. Enfoque UTE, 9(2), 125-137. https://doi.org/10.29019/enfoqueute.v9n2.252

Pauli, H., Gottfried, M., Lamprecht, A., Niessner, S., Rumpf, S., Winkler, M., Steinbauer, K., \& Grabherr, G. (2015). Manual para el trabajo de campo del proyecto GLORIA. Aproximación al estudio de las cimas. (5) 1-150. https://doi.org/10.2777/37575

Rodriguez, F., Oliet, J., Abellanas, B., Cuadros, S., Fernandez, P., \& Zornoza, R. (2004). Manual de ordenación de montes de andalucía (Consejería). 
https://www.juntadeandalucia.es/medioambiente/web/Bloques_Tematicos/Publicacion es_Divulgacion_Y_Noticias/Documentos_Tecnicos/manual_ordenacion_montes_anda lucia/manual_ord_montes.pdf

Santa Cruz, L., Cano, L., La Torre, M., Rodriguez, E., \& Campos, J. (2019). Inventario de la flora de angiospermas del distrito Pulán, provincia Santa Cruz, Cajamarca, Perú. Arnaldoa, 26(1), 139-212. https://doi.org/10.22497/arnaldoa.261.26108

Sklenár, P., \& Balslev, H. (2005). Superpáramo plant species diversity and phytogeography in Ecuador. Flora, 200(5), 416-433. https://doi.org/10.1016/j.flora.2004.12.006

Urgiles, N., Cofre, D., Loján, P., Maita, J., Albarez, P., Báez, S., Tamargo, E., Eguiguren, P., Ojeda, T., \& Aguirre, N. (2018). Plant diversity, community structure, and aereal biomass in a paramo ecosystem of Southern Ecuador. Bosques Latitud Cero, 8(1), 13. https://bibdigital.epn.edu.ec/bitstream/15000/20230/1/Diversidad de plant. SB 2018.pdf

Vistin, D., \& Barrero, H. (2017). Floristic study of the green forest always montanoof the community of Guangras, Ecuador. Avances, 19, 218-226.

http://suia.ambiente.gob.ec/documents/10179/346525/Estrategia+de+Sostenibilidad+F inanciera+del+SNAP.pdf/f1ef1719-5c4e-46a0-8ef5-49eb1c60a119 


\section{PARA CITAR EL ARTÍCULO INDEXADO.}

Vistin Guamantaqui, D. A., Muñoz Jácome, E. A., \& Ati Cutiupala, G. M. A. C. (2020). Monitoreo del Herbazal del páramo una estrategia de medición del cambio climático en la Reserva de Producción de Fauna Chimborazo. Ciencia Digital, 4(2), 32-47. https://doi.org/10.33262/cienciadigital.v4i2.1195

\section{¿ Ciencia}

El artículo que se publica es de exclusiva responsabilidad de los autores y no necesariamente reflejan el pensamiento de la Revista Ciencia Digital.

El artículo queda en propiedad de la revista y, por tanto, su publicación parcial y/o total en otro medio tiene que ser autorizado por el director de la Revista Ciencia Digital.
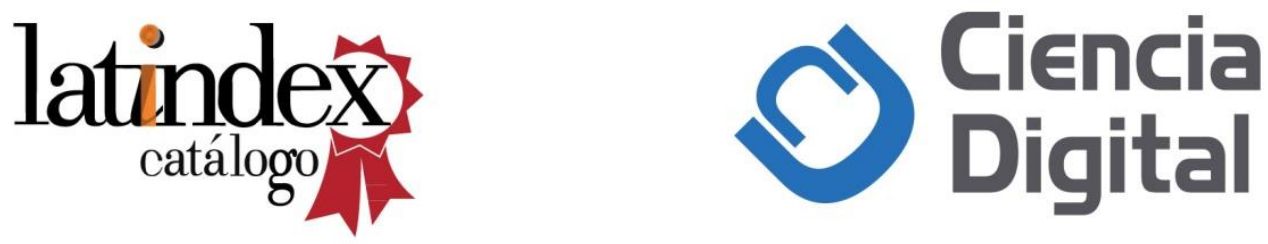